\title{
Procesos de individualización en contextos culturales diferentes: los adolescentes en México y Alemania
}

\author{
Individualization processes in different cultural \\ contexts: adolescents in Mexico and Germany
}

\author{
doi: http://dx.doi.org/10.32870/ \\ espiral.v23i67.4309
}

\section{Resumen}

Este artículo explora las principales distinciones que marcan los procesos de individualización de dos grupos de adolescentes de diferentes contextos socioculturales, ubicados en Guadalajara (México) y Bremen (Alemania). El estudio explora algunas particularidades en el proceso de individualización que adquieren significado a la luz de las condiciones y los contextos culturales en los que los jóvenes desarrollan sus vidas. El enmarque teórico lo conforman conceptos sociológicos propuestos por Beck, Giddens y Lash (1997) que aluden a la modernidad reflexiva e individualización como referentes orientadores para comprender la construcción de las identidades juveniles y las distintas formas en las que los adolescentes se adhieren hoy a la sociedad. El diseño metodológico fue el estudio de caso con unidades de análisis incrustadas (Yin, 1994), utilizando la entrevista como instrumento principal de recolección de datos.

Palabras clave: identidad, individualización, adolescentes, modernidad reflexiva, cultura.

\section{Gloria Briceño Alcaraz*}

\begin{abstract}
This article explores the major distinctions that mark the process of individualization of two groups of teenagers from different socio-cultural contexts located in Guadalajara (Mexico) and Bremen (Germany). The study explores some peculiarities in the process of individualization that acquire meaning in the light of the conditions and cultural contexts in which young people develop their lives. The theoretical framing formed the sociological concepts proposed by Beck, Giddens, Lash (1997) that refer to the reflexive modernity and individualization as guiding reference for understanding the construction of youth identities and the different ways in which adolescents today stick to the society. The methodological design was the case study with embedded units of analysis (Yin, 1994) using the interview as the main instrument of data collection.
\end{abstract}

Keywords: identity, individualization, adolescents, reflexive modernity, culture.

\footnotetext{
- Profesora-Investigadora del Centro de Investigaciones Pedagógicas y Sociales de la Secretaría de Educación Jalisco (SE)). evora@prodigy.net.mx

Fecha de recepción: 03 de noviembre de 2015. Fecha de aceptación: 06 de abril de 2016.
} 


\section{Introducción}

En este artículo se recuperan algunos aspectos de una investigación cualitativa realizada con adolescentes de secundaria pertenecientes a contextos socioculturales diversos, pero altamente urbanizados, como son Guadalajara (México) y Bremen (Alemania). Cabe mencionar que el trabajo de campo en Bremen se realizó en 2012 durante una estancia de investigación de tres meses que contó con el apoyo del Servicio Alemán de Intercambio Académico (DAAD, por sus siglas en alemán). Por su lado, los datos del grupo de adolescentes mexicanos provienen de un estudio diagnóstico más amplio efectuado con anterioridad para configurar cartografías del consumo cultural (lectura, cine, televisión, internet, bibliotecas, etc.) de estudiantes de secundaria del sector público en el estado de Jalisco (Villalpando, et al., 2012).

Por lo tanto, se abordarán algunos aspectos de la individualización juvenil (específicamente, en la etapa adolescente) que guardan relación con las expectativas que los jóvenes tienen sobre la escuela, sus proyectos para la vida adulta y las opciones de realización que visualizan a partir de las condiciones de sus respectivos ambientes.

Se partió del supuesto de que los adolescentes de hoy día se enfrentan al gran desafío de lograr la conformación de su identidad en condiciones de cambios vertiginosos, inestabilidad y riesgo social, que son características de las sociedades urbanas modernas (Beck, 2002; Brater, 2002). En ese proceso de búsqueda individual, las condiciones y agentes, en sus respectivos contextos, desempeñarán funciones sustentadoras de tal tarea que les facilitarán a los jóvenes el camino al logro de la identidad. 


\section{El sujeto: actor y malabarista de su propia autobiografía}

De acuerdo con Beck, Giddens y Lash (1997), el tránsito de la sociedad industrial (primera modernidad) a la actual etapa llamada "modernidad reflexiva" (segunda modernidad) transformó sustancialmente la relación individuosociedad debido a diversos procesos de reorganización social, política y económica acaecidos en la sociedad occidental a partir de los años sesenta del siglo pasado (entre ellos, la debacle de los regímenes socialistas y la posterior recomposición del sistema sociopolítico mundial en nuevas hegemonías supranacionales, el abismo "Norte-Sur", la globalización y la revolución de las TIC).

Se argumenta que la sociedad industrial fue el sustrato social de la primera modernidad, en la que los marcos discursivos y normativos legitimaban las instituciones más representativas: Estado-nación, escuela, Iglesia, familia y clase social, a cuyo amparo los individuos se incorporaban casi de manera automática a la sociedad por medio de los roles sociales preestablecidos por esta. Se dice que tal época estuvo marcada por una lógica de "estructuras", a diferencia de la actual modernidad, donde los "flujos" son la constante (Lash, 2003).

Con la transición a la modernidad reflexiva, la vida de los individuos sufrió un gran cambio que se reflejó en sus prácticas, estilos de vida y relaciones:

Tanto en la política como en el ámbito científico o en la vida cotidiana, con harta frecuencia ha dejado de estar claro quién o qué constituye la familia. Los límites se hacen borrosos, las definiciones vacilantes; crece la inseguridad (Beck-Gernsheim, 2000, p. 13).

Ante ese panorama de incertidumbres que va de la esfera privada a la pública, los individuos están forzados a desarrollar una conciencia reflexiva que está alerta ante los 
múltiples riesgos generados en la sociedad (tecnología sin control, alimentos cancerígenos, deterioro del ecosistema, criminalidad, violencia pública y corrupción política):

Los riesgos se han convertido en una de las principales fuerzas de movilización política, sustituyendo muchas veces, por ejemplo, a las referencias a las desigualdades asociadas a la clase, la raza y el género. Esto subraya el nuevo juego de poder del riesgo y sus metanormas: en una época de incertidumbres fabricadas, ¿quién tiene que definir los riesgos de un producto, de una tecnología y con qué criterios? (Beck, 2002, p. 6).

Estas condiciones azarosas se combinan para conformar un sujeto incrédulo de las instituciones que ya no le dan respuesta a sus necesidades de dirección, no obtiene certeza de ellas, por lo que tiene que ser él mismo quien como "actor, diseñador, malabarista y director de escena de su propia biografía, identidad, redes sociales, compromisos y convicciones" (Beck, Giddens y Lash, 1997, p. 205) se conforme en este tipo de sociedad posmoderna. Es entonces que surge la categoría de individualización como intento de comprender al sujeto en su soledad ante la tarea inacabada de conformarse como individuo (Beck, Giddens y Lash, 1997).

Habría que puntualizar que la individualización no tiene que ver con el individualismo institucionalizado de la propiedad privada, tampoco con un sentido liberal del libre mercado ni de "ser individuo" en el sentido de la Ilustración, sino que, por el contrario, alude a un nuevo sujeto que se construye por sí mismo, que se hace individuo, en medio de las condiciones volátiles e inseguras que imprime la globalización en todos los órdenes de la vida y que impactan la manera en cómo se construyen los proyectos de vida y, por ende, las identidades (Beck y Beck-Gernsheim, 2002).

Si estas son las principales coordenadas en las que se reorganizan los "flujos" en esta nueva sociedad globalizada, 
surgen varias preguntas con relación a las subjetividades juveniles y sus contextos de origen: ¿cómo podemos comprender las diferencias y similitudes en los procesos de individualización que ocurren en contextos socioculturales diversos? ¿Qué papel juegan las instancias y agentes socioculturales en la construcción de las identidades en ambientes específicos del desarrollo? Y ¿cómo enfrentan los adolescentes de diversos contextos los desafíos de conformarse en individuos?

\section{Antecedentes}

La etapa de la adolescencia ha sido definida como la más vulnerable, dados los numerosos cambios que ocurren simultáneamente en la génesis del desarrollo psicosocial e intelectual de los individuos entre los doce y diecinueve años. Durante el corto tiempo en que transcurren estos cambios estructurales y funcionales, los jóvenes están sometidos a una presión considerable, ya que tienen que resolver la crisis de identidad y tomar decisiones importantes para su vida futura (Papalia, et al., 2010).

Tener una perspectiva de vida a futuro, un trabajo fijo, o bien continuar con los estudios dependerá de varios factores socioeconómicos y culturales que se conjugan en torno al sujeto adolescente en el proceso de conformarse como individuo conquistando una identidad social.

Sabemos por la literatura científica anglosajona que la individualización pone a prueba las capacidades de los jóvenes para desarrollar sus proyectos de vida en condiciones azarosas como las actuales.

En Alemania, por ejemplo, se cuenta con un sistema "dual" de aprendices, en el cual los adolescentes que optan por un oficio pueden asistir la cuarta parte del tiempo a la escuela y el resto de la semana son capacitados en la industria o fábrica, donde reciben un entrenamiento remu- 
nerado por la propia empresa-tutor hasta el término del entrenamiento. Los egresados son evaluados, y aquellos que obtienen los mejores puntajes son seleccionados para quedarse en la empresa bajo un contrato laboral seguro.

Ese sistema de formación dual ha tenido gran éxito durante décadas, pues ha evitado la deserción escolar y el desempleo juvenil, ya que la industria, en sus diferentes sectores, absorbe la mano de obra calificada de jóvenes que cursan el nivel escolar intermedio. Sin embargo, la situación en el caso de los jóvenes mexicanos es muy distinta, pues lo que prima en este nivel escolar es la deserción; inclusive se diría que el sistema educativo desconoce con qué capacidades y estrategias se enfrentan estos estudiantes para conformar sus proyectos personales (Brater, 2002; Reyes, 2009).

Precisamente, el asumir compromisos consigo mismo y la sociedad, así como configurar un proyecto personal, son condiciones para el logro de la identidad adulta, según algunos psicólogos (Kroger, 2003; Marcia, 1993). Sin embargo, cada vez más se observa en los jóvenes un fenómeno llamado "moratoria", que retrasa ese momento, al menos en las sociedades urbanas occidentales (Papalia, et al., 2010).

$\mathrm{Al}$ respecto, hay coincidencias en señalar tal tendencia entre los jóvenes estadunidenses de clase media, para quienes "la adultez" representa una "opción" o estilo de vida, a diferencia de sus padres, que veían en esta un estado de crecimiento que traía consigo diversos compromisos como casarse, tener hijos, comprarse una casa, etc. (Twenge y Campbell, 2009). Esta situación contrasta con lo que buena parte de los jóvenes mexicanos esperan lograr en esa etapa: ciertos satisfactores en el ámbito laboral, mejorar el nivel de vida, tener una profesión, establecer una familia y, en suma, tener un proyecto personal (Instituto Mexicano de la Juventud, 2012). 
$\mathrm{Al}$ respecto, varios autores han señalado que el desajuste entre las nuevas condiciones estructurales producidas por la globalización y la falta de oportunidades de trabajo para los jóvenes en diversos contextos urbanos modernos diversificó la manera como estos se incorporan a la sociedad. Eso ha llevado a la escuela a una actualización de sus métodos de enseñanza, incorporando las TIC como estrategias para el aula, pero también a considerar otros recursos educativos formales e informales que les ayuden a adquirir nuevas competencias (Beck, 2002; Tiramonti, 2004; Morduchowicz, 2004).

Efectivamente, se sabe que, por recomendación de los organismos internacionales (UNESCO, OCDE), los sistemas educativos globales han hecho cambios en los programas de enseñanza-aprendizaje para evitar que queden obsoletos ante el advenimiento masivo de las TiC. Sin embargo, tendría que ser el papel social estructural de la escuela el que fuera replanteado pues, como señalan algunos críticos,

La escuela sólo puede tener algo que ver con la vida de forma muy puntual -por ejemplo, en el dominio de la transmisión cognitiva de determinados contenidos funcionales del saber-, mientras que su significación como instancia social, con tareas efectivas desde el punto de vista evolutivo, prácticamente desaparece (Brater, 2002, p. 135).

En el contexto del presente estudio, se sabe por las encuestas que la escuela sigue siendo percibida como una condición básica para conseguir un buen trabajo, es decir, grandes sectores de la población la legitiman como transmisora de saberes. ${ }^{1}$ Habría, sin embargo, que tamizar esa información, ya que para una parte de los adolescentes de secundaria la

I. Según la Encuesta Nacional de Valores en Juventud (Instituto Mexicano de la Juventud, 20I2), ocho de cada diez jóvenes entrevistados piensan que la educación se traduce en éxito, $53.5 \%$ considera que con educación podrá conseguir un buen empleo, y el $17.6 \%$ afirma que le ayudará a alcanzar un buen desarrollo profesional. 
escuela no es condición sine qua non para alcanzar el éxito profesional y tener mejores oportunidades en la vida; por el contrario, es justamente en dicho nivel de la educación básica que un gran porcentaje de alumnos desertan por razones económicas y por no encontrar su vinculación con la vida laboral (Ayala, 2001; Meza y Pederzini, 2008; Briceño, 2011).

Otras investigaciones indican que si bien los adolescentes mexicanos provenientes de la clase media tienen más probabilidades de continuar en la escuela, otros tantos de contextos marginales urbanos, semiurbanos y rurales la abandonan para enrolarse en actividades del mercado informal, o bien, para irse al "Norte" (Meza y Pederzini, 2008; Villalpando, et al., 2012).

\section{La individualización como proceso hacia la identidad}

La investigación aquí referida respondió, por una parte, a la necesidad de complementar con miradas interpretativas los estudios tipo encuesta (descriptivos) que generalmente se realizan en el ámbito de estos estudios para conocer las tendencias juveniles en cuanto a salud, educación, consumo, etc. Si bien los datos empíricos son necesarios para ofrecer una panorámica o diagnóstico sobre cierto sector de la población, ese tipo de metodologías resultan insuficientes para indagar con profundidad en los procesos de socialización e identidad que tienen su origen en la conformación de la subjetividad (Ruiz y Briceño, 2005).

Por otra parte, hay una inquietud metodológica por conocer hasta qué punto la categoría individualización puede explicar los procesos de socialización y conformación de las identidades juveniles en el contexto mexicano.

Si bien ese concepto arroja una nueva mirada sobre la recomposición subjetiva de los jóvenes frente al debilitamiento de las instituciones de manera global, habría 
que recordar que la categoría no ha sido suficientemente trabajada en poblaciones latinas; por lo tanto, resulta relevante analizar su connotación descriptiva en los contextos sociodemográficos asimétricos que configuran el "abismo" Norte-Sur, como son, en este caso, Bremen (Alemania) y Guadalajara (México). Eso podría contribuir a dotar el estudio del caso de validez empírica para describir con más precisión los entramados subjetivos a través de los cuales los adolescentes socializan y dan sentido a sus vidas.

Dado que la unidad de análisis son los procesos de individualización de los adolescentes que cursan la secundaria en dos contextos distintos, se eligió el estudio de caso para tratar de analizar las singularidades expresadas a partir de las entrevistas en concordancia con el contexto al cual pertenecen los sujetos, y poder así identificar e interpretar algunos elementos subjetivos comunes que conforman los mundos de vida al interior de su grupo.

Mertens (2005) define el estudio de caso como una investigación sobre un individuo, grupo, organización, comunidad o sociedad, que es visto y analizado como una entidad: un todo en relación con su contexto. El estudio de caso es de tipo instrumental por su finalidad, pues se pretende que los hallazgos aporten a la construcción y refinación del concepto teórico de individualización (Stake, 2000).

Se convino en segmentar la unidad de análisis principal arriba comentada en "unidades incrustadas" o subunidades con la finalidad de posibilitar la contrastación intergrupal de los significados y poder analizar las convergencias y divergencias en las formas de socialización de ambos grupos (Yin, 1994).

Las unidades incrustadas para ambos grupos fueron: 1) capacidad de negociación o estrategias con las que los jóvenes enfrentan y se adaptan a las demandas de su entorno social; 2) agencia: búsqueda individual en contextos de crisis social; 3) representaciones: conjunto de informaciones, 
creencias, opiniones y actitudes sobre la escuela y su entorno social; y 4) capacidad reflexiva o reflexividad: utilización de fuentes de información para retroalimentar sus acciones y orientar sus decisiones.

Cabe resaltar que esta metodología no pretende establecer una comparación entre los sujetos en particular (desde la dimensión psicológica), sino, más bien, identificar patrones socioculturales al interior de cada grupo a partir de los cuales se puedan establecer ciertas categorías en un meta-análisis y así trazar posibles vínculos entre ambos grupos en cuanto a los procesos de individualización que median en esta etapa juvenil.

\section{Los dos grupos participantes}

En esta investigación participaron en total veintidós adolescentes de clase media, en edades entre doce y diecisiete años, tanto de Guadalajara, México (grupo 1) como de Bremen, Alemania (grupo 2). Cada grupo estuvo conformado por once estudiantes de secundaria (cuatro hombres y siete mujeres). Las entrevistas fueron realizadas en su mayoría en secundarias del sistema público estatal, tanto en Bremen como en Guadalajara.

Se entrevistó a los adolescentes con base en un instrumento que contenía diez preguntas temáticas detonadoras acerca de diversos aspectos de la vida cotidiana, tales como pasatiempos, escuela, interacciones sociales y afectivas, expectativas y proyectos. De igual manera, se recabó información complementaria con ciertas autoridades y representantes de instituciones en la ciudad de Bremen (directores, maestros, padres de familia). ${ }^{2}$

2. La sra. Regine Komoss, coordinadora del Centro de Formación Docente de la Universidad de Bremen, la sra. Barbara Suchland, directora del Centro de Terapia Pedagógica “Alten Eichen”, y el director de la secundaria general Gesamtschule 
Las ubicaciones geográficas de los adolescentes participantes en este estudio se muestran en la Imagen 1 e Imagen 2:

Imagen 1. Ubicación de Bremen, Alemania

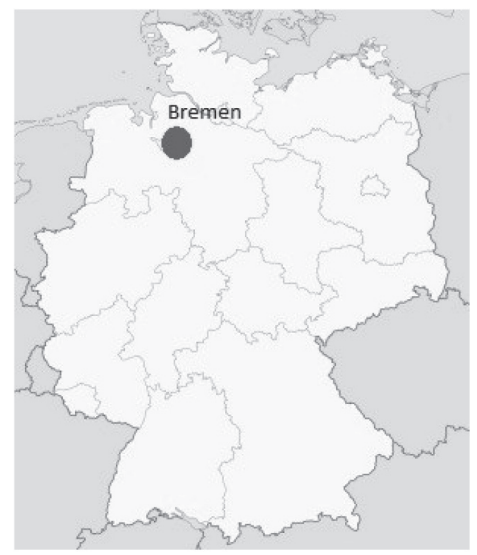

Fuente: Wikipedia (s/fa).

Imagen 2. Ubicación de Guadalajara, México

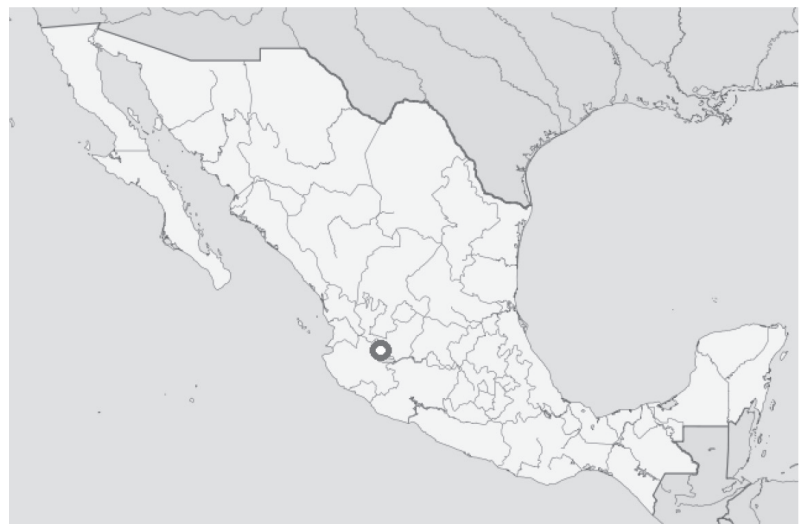

Fuente: Wikipedia (s/fb).

"Sportzentrum an den Ronzelenstrasse", lugar donde se realizaron la mayor parte de las entrevistas. 
No solamente la situación geográfica separa a estos dos contextos (entre Guadalajara y Bremen existe una distancia de $9563 \mathrm{~km}$ en línea recta), sino también el idioma, la cultura y siete husos horarios de diferencia. Se presentan a continuación algunos datos sociodemográficos que contextualizarán los escenarios donde estos adolescentes se desarrollan.

\section{I. Grupo I: el contexto mexicano}

En México, por cada 1000 habitantes hay diecinueve nacimientos (18.41\%). La población juvenil (de doce a veintinueve años) representa el $32.1 \%$ de la población total; es decir, en números absolutos son treinta y siete millones de jóvenes de ambos sexos, pero se estima que este bono demográfico para el país irá descendiendo drásticamente en las siguientes décadas.

De ellos, el 56\% (un poco más de la mitad) recibe educación media superior, en comparación con el 84\% logrado en promedio en el resto de los países de la OCDE. La problemática para este grupo es insertarse en el mercado de trabajo: siete de diez jóvenes utiliza las redes informales para poderlo hacer. La tasa de desempleo entre jóvenes de catorce a veintinueve años se estima en 53.2\% (Diario Oficial de la Federación, 2014; OCDE, 2013).

Los participantes de este grupo se ubican en la ciudad de Guadalajara y su zona metropolitana. Los entrevistados están inscritos en diferentes secundarias tanto públicas como privadas, y los padres cuentan con formación profesional e incluso algunos con estudios de posgrado, desempeñando sus actividades en el sector profesional de la educación y de servicios. Se puede decir que en el caso de este grupo hay un capital cultural familiar medio-alto, evidenciado en el grado de instrucción del jefe de familia y reflejado en el equipamiento y distribución de los bienes culturales en el hogar (biblioteca familiar, dispositivos elec- 
trónicos variados e internet de banda ancha). En general, se registra que los bienes u objetos de entretenimiento (televisión, reproductor DVD, laptop, tablet, etc.) son poseídos en mayor proporción que otros derivados de otras expresiones artísticas (pinturas, esculturas y colecciones de arte), independientemente del tamaño de la localidad en donde se localizan las viviendas (Villalpando, et al., 2012).

En el estudio realizado sobre Consumo y cambio cultural en estudiantes de secundaria en Jalisco (Villalpando, et al., 2012) se evidenció que los medios audiovisuales disponibles en el hogar (televisión, radio, reproductor DVD, etc.) forman parte de la vida cotidiana de las familias, independientemente del nivel socioeconómico al que pertenecen. En cuanto a las costumbres, creencias e ideologías, los jóvenes mexicanos priorizan en sus vidas la importancia de la familia, seguida por el trabajo, la pareja, el dinero y la escuela, todo esto a diferencia de un $60.7 \%$ para quienes la política carece de importancia.

En cuanto a la tolerancia hacia parejas del mismo sexo, cuatro de diez jóvenes (44\%) se declararon de acuerdo en este tipo de uniones, mientras que un $33.7 \%$ en desacuerdo; en este mismo, tema un $47.8 \%$ está en desacuerdo con que parejas de homosexuales adopten un niño, ya que consideran que un hijo debe tener padre y madre para su sano desarrollo (Instituto Mexicano de la Juventud, 2012). Esto nos habla de una prevalencia y arraigo en costumbres propias de una sociedad aún tradicional, donde las instituciones juegan un papel regulador en la vida privada.

\subsection{Grupo 2: el contexto alemán}

Bremen está localizada al noroeste de Alemania y cuenta con 546451 habitantes. Esta ciudad conforma, junto con el puerto de Bremen (Bremerhaven), la Ciudad Libre Hanséatica del estado de Bremen, que forma parte de los dieciséis estados de la República Federal de Alemania. Bremen tiene 
diversos atractivos, entre los que se encuentran la antigua plaza del Mercado y el Ayuntamiento, con su bella fachada gótica y renacentista. En ese gran espacio, se levanta imponente la estatua más emblemática y motivo de orgullo de la población: Rolando, que representa precisamente la libertad hanséatica.

De actividad comercial y portuaria por tradición, en la actualidad Bremen es un referente industrial de gran importancia para la Unión Europea (UE) por tener en su territorio empresas del calibre de la Daimler-Chrysler y la EADS (European Aeronautic Defense and Space), que fabrica principalmente aeronáutica civil y militar, misiles y cohetes espaciales de alta tecnología mundial.

Entre los datos sociodemográficos más relevantes se encuentra que por cada 1000 habitantes hay ocho nacimientos (8.23\%). Uno de cada ocho alemanes cuenta con menos de quince años de edad; el porcentaje de este grupo es de $13 \%$ en Alemania, frente a un $28 \%$ en Latinoamérica y el Caribe, y un 26\% en el resto del mundo (Datenreport, 2014). El envejecimiento de la población alemana contrasta con los nacimientos de la población de origen inmigrante (principalmente de ascendencia turca), y en una década se prevé que serán los refugiados de origen sirio, quienes llegaron en el 2015 (rebasando el millón), quienes registren la mayor tasa de natalidad del país (Die Welt, 2013).

En general, las condiciones de los jóvenes en Alemania pueden ser consideradas como de las más favorables de Europa, ya que cuentan con un gran número de apoyos para su formación (financiamiento para el estudio, transporte subvencionado, seguro médico, etc.) que les permiten mantenerse mientras estudian la universidad, además, gracias al Sistema de Formación Dual pueden realizar una capacitación técnica e incorporarse a la vida laboral, evitando el desempleo. 
Esas circunstancias propician que los jóvenes se independicen tempranamente de sus padres, gracias al financiamiento llamado $B A f \ddot{o} G$ (Bundesausbildungsförderungsgesetz) al que todo estudiante universitario alemán tiene derecho, o bien, que puedan realizar estancias de estudio en otros países de la UE con becas Erasmus.

El problema del costo de vivienda lo resuelven al juntarse con amigos o compañeros de estudios en espacios compartidos (Wohngemeinde), mientras que los alimentos son proporcionados por una cuota reducida en los inmensos comedores universitarios, llamados "mensa". La gran movilidad, la multiculturalidad y la diversidad son rasgos característicos de las universidades alemanas que acogen a estudiantes de todo el mundo que aplican a sus programas de posgrado (Banco Mundial, s/f).

Dos de los acontecimientos más relevantes que contribuyeron a transformar la sociedad multicultural que hoy es Alemania fueron, sin duda, la caída del muro de Berlín y el subsecuente proceso de reunificación entre la República Democrática (Deutsche Demokratische Republik) y la Alemania Federal (Bundesrepublik Deutschland), hechos transcurridos entre 1989 y 1990.

En los integrantes de la generación del cambio -jóvenes que tienen alrededor de veinticinco años- difícilmente se puede encontrar parecido en costumbres, creencias y valores con sus antecesores. La juventud alemana es más abierta, tolerante y más relajada de lo que fueron sus padres y, aún más, sus abuelos. Ellos son la generación "multikulti” (multicultural), "verde" (con conciencia ecológica y vegetariana), que hace eco del lema de "igualdad, libertad y fraternidad" con valores que rigen la actual constitución y que ponen en práctica en los grupos de amigos, escolares, clubes y familia en donde conviven.

De los poco más de ochenta y dos millones de habitantes en Alemania, quince tienen un trasfondo migratorio (2.5 
millones principalmente de origen turco, y el resto de los países del Este). A estos se deben agregar los refugiados sirios que llegaron a fines del 2015 y que recibieron asilo político. Esta pluriculturalidad de sus habitantes obliga al Gobierno alemán a redoblar sus esfuerzos para integrar a los antiguos habitantes y a los recién llegados a través de sus políticas educativas, sociales, laborales y de medios de comunicación (televisión y radio).

La sociedad demanda al Gobierno que las políticas de integración sean también de asimilación, so pena de generarse disturbios sociales graves como los que hubo en Francia, por ejemplo, con la población juvenil de origen migrante. De ahí que el Gobierno alemán en los últimos años haya empezado a reforzar con urgencia sus políticas públicas hasta hacerlas cotidianidad en la cultura escolar (Deutschland Magazin, s/f).

Un dato cultural relevante que trasciende el ámbito escolar, pero que está muy ligado a este, es que Alemania sigue siendo un país de lectores. Ahí, el gusto por la lectura se incentiva desde edades muy tempranas en la escuela y en las propias familias. Esa práctica hace que los jóvenes en su mayoría prefieran leer un libro que sentarse horas frente a la televisión (el promedio de libros que leen estos jóvenes al año es de doce, mientras que en nuestro país es de 2.8, según la Encuesta Nacional de Lectura -Fundación Mexicana para el Fomento de la Lectura A. C., 2012-).

Los participantes de este grupo se ubicaban en la amplia franja de la clase media, y al menos cuatro de los once provenían de familias migrantes tanto de Europa del Este como de Asia Central. Ellos están integrados y asimilados a la sociedad alemana (tienen nacionalidad alemana y dominan el idioma). En Bremen se habla aún el dialecto Platt-deutsch entre los adultos mayores, pero los jóvenes prefieren el alemán alto (Hoch-deutsch) mezclado con alguna jerga de moda; no obstante, resulta interesante comprobar que cada 
vez más algunos grupos de rock alternativos de la localidad incorporan el Platt-deutsch en sus propuestas como parte de su identidad cultural regional.

En un país donde abundan las fundaciones, ONG y clubes orientados al servicio social, resulta comprensible que ocho de diez jóvenes estén involucrados en alguna de estas y destinen al menos cuatro horas a la semana en ese trabajo social no remunerado. Sus estilos de vida han cambiado, así como la forma de establecer la pareja: aunque la unión de personas del mismo sexo está reconocida jurídicamente, no es reconocida como "matrimonio" como en el resto de países europeos. Por otra parte, el hecho de que las parejas pospongan o declinen el proyecto de ser padres se debe, entre otras cosas, al costo que representan los hijos, la pérdida de libertad, y porque los hijos suelen ser incompatibles con la profesión (Schmaus, 2014).

Suele decirse que los jóvenes de la generación actual han recuperado el orgullo de sentirse alemanes con una nueva identidad, abierta, flexible y orientada a la tolerancia con los otros. Gran parte de este suceso ha sido producto de varios factores, como ser la principal potencia económica de la UE y de Occidente (aquí se producen casi el 30\% de los avances tecnológicos y científicos del mundo). Otro motivo de orgullo -no menos importante para los ciudadanos "de a pie"- es su selección de futbol, que ha conquistado el campeonato mundial un par de veces (y quedando siempre entre los tres primeros lugares).

\section{Análisis e interpretación de los resultados}

A partir de la sistematización y análisis de los datos, se construyó una matriz común de significados que integró cuatro categorías para ambos grupos; las distinciones más marcadas entre el grupo 1 y 2 se reflejan en la orientación 
que toman los procesos de individualización según el grupo y su contexto, como se puede ver en el Cuadro 1.

Cuadro 1. Matriz de análisis de los significados: adolescentes mexicanos y alemanes (2015)

\begin{tabular}{|c|c|c|}
\hline Categoría & Descripción & Orientación del proceso \\
\hline a) Negociación & $\begin{array}{l}\text { Maneras de establecer } \\
\text { acuerdos y compromisos } \\
\text { con los demás. }\end{array}$ & $\begin{array}{l}\text { (Gpo.1) Consensuado con los } \\
\text { padres. } \\
\text { (Gpo.2) Consensuado consigo } \\
\text { mismo, pares y familia. }\end{array}$ \\
\hline b) Agencia & $\begin{array}{l}\text { Búsqueda e iniciativa } \\
\text { individual para responder } \\
\text { ante desafios y problemas. }\end{array}$ & $\begin{array}{l}\text { (Gpo.1) Centrada en } \\
\text { componentes socio-afectivos. } \\
\text { (Gpo.2) Centrada en la } \\
\text { individualidad y racionalidad. }\end{array}$ \\
\hline c) Representaciones & $\begin{array}{l}\text { Significados y valores hacia } \\
\text { la escuela y la sociedad. }\end{array}$ & $\begin{array}{l}\text { (Gpo.1) La consideran } \\
\text { necesaria para su desarrollo. } \\
\text { Contacto social y afectivo con } \\
\text { pares. } \\
\text { (Gpo.2) Requiere esfuerzo y } \\
\text { disciplina para tener buenas } \\
\text { notas. Sinónimo de éxito en la } \\
\text { vida. Contacto social y afectivo } \\
\text { con pares. }\end{array}$ \\
\hline d) Reflexividad & $\begin{array}{l}\text { Maneras de utilizar fuentes } \\
\text { de información que guíen la } \\
\text { toma de decisiones. }\end{array}$ & $\begin{array}{l}\text { (Gpo.1) Aplicación parcial de } \\
\text { la información para beneficio } \\
\text { propio o para la toma de } \\
\text { decisiones. } \\
\text { (Gpo.2) Aplican la información } \\
\text { a experiencias concretas de la } \\
\text { vida cotidiana para su beneficio } \\
\text { y toma de decisiones. }\end{array}$ \\
\hline
\end{tabular}

Fuente: elaboración personal.

Cabe aclarar que esta clasificación es estática y no representa cómo en el plano vivencial o fenomenológico interac- 
cionan o se combinan entre sí los procesos; por ejemplo, quien demuestre aplicar la información en su vida cotidiana (reflexividad), también será capaz de responder los desafíos y negociar sus compromisos con los otros. Igualmente, la forma de resolver los problemas (apoyado en otros o de manera independiente) dependerá de su iniciativa personal o agencia.

A continuación, en los estudios de caso se describen y analizan fragmentos de los discursos de las entrevistas que aluden a una interpretación dinámica de los aspectos cognitivos y emocionales que les suscitaron a los entrevistados las preguntas. Se optó por partir de lo que la escuela les representa para de ahí tejer la red de significados y vinculaciones con las otras categorías.

\section{I. Caso I: los adolescentes de Guadalajara ${ }^{3}$}

Los estudiantes de este caso visualizan sus proyectos partiendo del placer que les da una actividad determinada considerada ahora como un hobby, pero que puede llegar a convertirse en una profesión; tienen una visión más amplia del mundo de la que tuvieron sus padres y por consiguiente modelos de identificación menos tradicionales. El "bombardeo" constante del marketing, del internet (redes sociales) y demás medios de comunicación les ofrece una variedad de personajes, modelos e íconos de quienes imitan sus maneras de hablar, de caminar y vestir.

Así, los adolescentes intentan a manera de ensayo y error construir una identidad propia e independiente de la impuesta por la familia, aunque a la sombra y respaldo de la misma. Su búsqueda está alentada más por referentes externos y socio-afectivos que racionales: consideran necesarios a los amigos y a un afán de independencia y exploración

3. Los extractos que aparecen en este apartado fueron obtenidos de la investigación Consumo y cambio cultural en estudiantes de secundaria en Jalisco (Villalpando, et al., 2012). 
más allá de su contexto habitual. Son adolescentes ávidos de conocer activamente su mundo, y eso implica, casi siempre, tener experiencias variadas, como lo reflejan sus voces:

Quiero seguir estudiando algo que tenga que ver con ingeniería en audio o algo así. Quiero enfocarme en creación de audio también [...] hacer un cuarto acústico con bocinas. Me gustaría saber posicionar todo en la música [...] mi inspiración es David Guetta, dj. Esto es mi hobby, pero le estoy invirtiendo ahorita de las bocinas y todo eso [...] ya lo estoy viendo para grande, para futuro (Villalpando, et al., 2012, p. 99).

Pienso estudiar arquitectura, hago dibujos y eso me agrada [...] otra cosa que hago son malabares de fuego, ahí con mis amigos, eso de dar presentaciones gratuitas para ayudar al colegio o estar ahí ayudando a los profes de que "oye, ayúdame con este friso, ayúdame a organizar esto, o cuídame tantito aqui", eso siempre me ha dado buenos puntos [...] siempre trato de ser muy unido a la familia y con mis amigos ser más solidario, entonces trato de llevármela bien con todos, me veo así como trabajador. También quisiera viajar (Villalpando, et al., 2012, p. 99).

Yo pienso que quiero ser psicóloga o estudiar ciencias de la comunicación, una de esas dos [...] me imagino más como mi mamá, así dando conferencias y eso, pero por otra parte me imagino estando en la tele[visión], ahí también, con algo de psicología, no sé, dando o dirigiendo un programa (Villalpando, et al., 2012, p. 98).

Pues me imagino como toda una gimnasta; el sueño sería como llegar a los olímpicos y ganarlos, competir mucho, ser la mejor, se puede decir, ese sería mi sueño (Villalpando, et al., 2012, p. 99).

Estas "viñetas" muestran diferentes ángulos de cómo se va gestando un proyecto de vocación o de vida futura y de cómo, a partir de la experiencia y exploración, los adolescentes van encontrando referentes significativos, sea a partir de 
modelos tradicionales o de otros que no lo son tanto, pero que están presentes en sus mundos de vida.

Las expresiones "yo pienso", "me imagino", "me gustaría", "mi sueño" los posicionan como actores con cierta agencia y consciencia reflexiva sobre el tema de su propia vida y lo que de ella quieren hacer. La posibilidad de llegar a ser $d j$, gimnasta, arquitecto, comunicadora o psicóloga les permite visualizar un plano de la realidad social que proyectan al futuro, lo que les ayudará a situarse imaginativamente en una realidad concreta cuando sean adultos. Por el momento no importa qué tan alcanzables sean sus sueños, lo que importa es que desde ahora son capaces de imaginarse haciendo una función social en el mundo que viven (agencia); es entonces que la escuela cobra un sentido, un para qué:

La verdad la escuela es lo básico porque no sería lo mismo estar trabajando en eso de dj sin estudiar; como que estudiando aprendes más y te haces de más amistades. Aparte iqué prefieres: que te llegue un inculto que sabes que lo puedes chantajear, o que te llegue una persona muy culta? Para mí, la carrera es importantísimo [sic] porque te puede durar para toda la vida (Villalpando, et al., 2012, p. I00).

Siempre me ha dado mucha flojera ir a la escuela, siempre, no sé, me da mucha flojera [...] creo que todo mundo va porque sus papás les dicen "tienes que ir" y porque desde chiquito te dijeron "tienes que ir a la escuela", y ya tienes la mentalidad. Pero, por otra parte, cuando veo a gente que no estudió -porque conozco a una amiga que la corrieron y ahora va a estudiar la secundaria abierta, que es un caos- pues sí como que yo no quiero vivir tan mal, debería estudiar, entonces, por eso es algo importante, no quieres hacerlo, pero tienes que hacer[lo] y ya (Villalpando, et al., 2012, p. 100). 
La tensión entre dos mundos contrastantes y por momentos antagónicos se hace evidente aquí. Por una parte, está el deseo de libertad para seguir explorando sin coerción su mundo, y por otra, la imposición del mundo adulto (la escuela) que frena sus anhelos. La resolución que los adolescentes están obligados a hacer los lleva a una negociación entre lo que pueden perder si la abandonan y las ganancias secundarias que les deparan estando en ella (los amigos).

En el despliegue imaginativo de sus proyectos de vida, se entremezclan la crítica, la preocupación y el deseo por mejorar las condiciones sociales y políticas del país; ello podría ser resultado de un cambio en la orientación de los procesos subjetivos reflexivos propios de la individualización juvenil, ya que ver hacia el futuro inmediato significa posicionarse como actores sociales que evalúan las condiciones de su entorno, así como las acciones de los otros (familia, instituciones, Gobierno, etc.) para luego tomar decisiones:

En México, para los chavos -digo, donde sea, el que le busca, le encuentra- sí la veo difícil últimamente. Veo a mis papás: se soban el lomo para darnos de comer a todos y para que tengamos buena educación. Pero veo los sistemas medios feos, ¿no?, desde los sistemas de salud, sistemas de educación, muchísima corrupción, y eso no me agrada, de hecho, me ofrecieron ahora que fui a Madrid casa allá, unos amigos de un tío, y que si quería ir a estudiar la carrera allá me daban casa, sería muy buena idea, pero pues tengo tres años para decidir. México me gusta mucho, su gente, su cultura, pero últimamente los sistemas han estado feos y eso no me agrada, ¿no?, pero, por otra parte, ¿qué hago? (Villalpando, et al., 2012, p. 104).

Tengo una materia que es "estudio de la ciudad", [en la que] fuimos a ver la ciudad y entonces me detuve a verla, ¿no?, tiene algunas partes que todavía se pueden salvar, pero está toda rayoneada, como grafiteada [sic]. También ves en cada esquina un borracho, pero es que ya ni siquiera existe eso de que "me voy a ir al campo a descansar" porque 
iya no existe! [...]. Aparte tenemos un pésimo Gobierno y una pésima economía, faltan empleos, entonces es como si todo fuera ilógico [...] jiteralmente el Gobierno es una basura! [...] Creo que México se puede salvar por la diversidad y todas estas cosas, pero finalmente hay un problema, bueno, muchos problemas que son como esto del narcotráfico. No creo que legalizar la droga sea una solución, va a ser lo mismo que con el alcohol y van a terminar tomando medidas como bien locas [sic] y te van a terminar metiendo como siempre a la cárcel (Villalpando, et al., 2012, p. 103).

Estas viñetas muestran las emociones ambivalentes que les suscita la realidad que viven en su contexto y país, pero expresan finalmente las preocupaciones vitales que ven sobre sus proyectos de vida (“¿Qué hago?”, se preguntan). Si bien por una parte valoran la cultura y las raíces propias, por la otra advierten que hay circunstancias adversas y un ambiente de riesgos para llegar a lograr sus metas. No obstante, en medio de la desesperanza de vivir entre "un pésimo Gobierno" y "sistemas feos" donde abundan la corrupción y la criminalidad, se rescata la diversidad de la cultura propia, que hace placentero vivir en México.

\subsection{Caso 2: los adolescentes de Bremen}

Para los miembros de este grupo, la negociación consensuada consigo mismos, con los pares y los otros es una forma de interacción común para conseguir sus propósitos, si no de manera total, al menos parcialmente, demostrándose de esa manera una búsqueda individualista y racional de resolver sus propios problemas.

Se puede advertir que en la base de la negociación se encuentra el diálogo para encontrar afinidades y conseguir acuerdos sociales. La orientación hacia el diálogo que enfatizan estos adolescentes es la voz internalizada de las políticas de integración educativa que el Gobierno difunde a través de los programas escolares y que hacen hincapié en 
los valores democráticos, en desmontar prejuicios y practicar la tolerancia con los otros: ciudadanos de distintas procedencias, de etnias, de culturas y religiones ajenas a la propia.

Esta es una medida precautoria que se entiende perfectamente al ser Alemania un país que sufrió las duras consecuencias de un régimen totalitario como el nacionalsocialismo en el siglo pasado, y que hoy es un crisol de culturas. Por ello, es prioridad del Estado alemán fomentar una plataforma de convivencia y tolerancia social donde la negociación sea la única forma de dirimir las pequeñas y grandes diferencias entre los ciudadanos.

En sus respuestas, se evidencia el tejido multicultural de su procedencia y el valor positivo que para ellos tiene la cultura de sus padres:

Yo diría que la cuestión multicultural no se ve en todas partes tan marcadamente como en la ciudad de Bremen, en la parte céntrica [...] yo crecí en un ambiente y familia multicultural, y por eso me parece muy bien, no tendría nada en contra de ello. Mi papá es de Dinamarca, por eso el apellido danés; mi familia, tíos, tías, tienen lazos con gente de Checoslovaquia, Dinamarca y España (entrevista personal, 2012a).

Mi mamá me dice que de pequeña hablaba solamente ruso y después en el kínder empecé a aprender el alemán y ahora solamente me comunico en alemán [...] el ruso lo entiendo en gran parte, pero no lo puedo hablar. Me agrada decir que tengo no solamente raíces alemanas, sino también de otro lado por parte de mi mamá [...] la cultura donde se formó ella (Uzbekistán) es más estricta que la alemana, por eso pienso que mi mamá me formó bien (entrevista personal, 20I2b).

La multiculturalidad en Alemania me parece muy bien, pues trae a Alemania mucho dinero, y seguramente sin la gente de otros países que viene a trabajar Alemania no podría sobrevivir. En mis planes para el futuro está viajar mucho fuera de Europa: Túnez, Tailandia, por la 
región de Asia [...] quizás luego al Caribe, pero no estoy muy seguro. Me parece muy interesante la cultura de Asia (entrevista personal, 20I2c).

En lo que se refiere al valor y sentido que le atribuyen a la escuela, se encuentran nuevamente formas de reflexividad características de una sociedad que promueve la individualización de los sujetos. Los adolescentes reconocen los conocimientos transmitidos por la escuela, pero destacan, sobre todo, las experiencias enriquecidas que proporciona este espacio para el desarrollo personal:

La escuela me ha hecho ser más consciente, pensar con mayor claridad sobre mí misma y mi entorno, ser lo que soy [...] todo lo que me ha interesado conocer, de física, por ejemplo, la escuela me ha proporcionado la información [...] y, lo importante, me ha mantenido informada y con curiosidad. Mis valores en mucho vienen de la educación en mi familia, pero también de la escuela, al menos de la forma en que mi escuela [Waldorfschule] nos favorece a pensar con apertura hacia la vida, en aceptar a los otros sin prejuicios [...] eso, creo, proviene de mi escuela Waldorf (entrevista personal, 20I2d).

La escuela me ha dado mucho conocimiento, aunque no sé exactamente en qué, pero sé ahora más que antes [...] sobre todo he aprendido en la escuela cómo relacionarme con los demás, cómo comportarme en la sociedad (entrevista personal, 20I2e).

La escuela me ha dado conocimientos, pero también me ha hecho una persona consciente de mí misma [...] claro que la recomiendo, porque uno aprende siempre para la vida adulta (entrevista personal, 20I2f).

Estas concepciones parecieran contradecir el supuesto "declive" de la escuela como instancia preferente para transmitir valores - de convivencia, tolerancia, normas sociales-, formas de vida y aprendizajes en la sociedad occidental (Dubet, 2006; Brater, 2000). Sin embargo, debe recordarse 
que la clase media (a la que pertenecen estos jóvenes) es la clase social que más se identifica con los valores y contenidos que la escuela promueve a través de sus planes de estudio.

También cabría destacar que en los alemanes la representación del éxito en la sociedad pasa por un sentido alto de competitividad y de esfuerzo personal (agencia individualista), lo que se traduce en obtener buenas calificaciones y de paso tener "buenos contactos" o "casarse con un rico" como estrategias para encontrar los mejores trabajos y una posición económica holgada en la vida adulta, aunque estas respuestas no han sido tan usuales en el grupo 2 del estudio, considerando el desarrollo y autosuficiencia que ha alcanzado la mujer en la sociedad alemana desde el siglo pasado.

Sin embargo, algunos analistas señalan que entre algunas adolescentes existe una especie de moda de regresar a ciertos cánones tradicionales de género (casarse, tener hijos, optar por el rol de ama de casa). Se comenta también que la generación actual prefiere trabajar y esforzarse menos que sus abuelos, pero viajar más, en contraposición con las características del pueblo alemán (trabajo, esfuerzo y disciplina) que hicieron posible el milagro económico después de la Segunda Guerra Mundial.

Si una persona quiere tener éxito, debe ser ambiciosa y trabajar mucho $[\ldots]$ algunos compañeros son así [...] pero no todos. Yo, por ejemplo, creo que no soy así [...] digo, si cumplo con mis deberes de la escuela, pero creo que soy más bien un poquito floja [ríe]. Mis calificaciones generalmente son treses [uno es la mejor nota y seis es la peor] (entrevista personal, 20I2f).

Para llegar a ser exitoso en la sociedad, se debe contar con buenos contactos. Yo, por ejemplo, pensaría en ascender rápidamente por medio de mis contactos, o bien, entrando en empresas jóvenes que no tengan restricciones y cuando crezcan, si se tiene suerte, esperar una 
oportunidad para ascender [...] y bueno, conseguir buenos trabajos, eso creo yo (entrevista personal, 20I2c).

Hay que hacer lo que a uno le interesa y le gusta, eso es importante, lo que a uno le da alegría hacer, entonces en eso se debe mantener y ser productivo (entrevista personal, 2012d).

Para que una persona alcance en esta sociedad el éxito rápido tiene que terminar la escuela con buenas notas o casarse con alguien rico [ríe]. ¡Yo quiero hacer las dos cosas! [ríe] (entrevista personal, 2012g).

Para tener éxito en la vida creo que uno debe aprender mucho, trabajar mucho para lograrlo, solamente así se puede salir adelante (entrevista personal, 2012e).

El "éxito en la vida" se inicia para estos adolescentes en el trabajo escolar; resulta interesante observar que a mayor necesidad de logro, mayor es la confianza que muestran en su sociedad. Tal confianza está respaldada por una estructura social que les garantizará trabajo y un sustento más que digno cuando terminen sus estudios. Dentro del país, o en otro de la UE, podrán desempeñar su actividad profesional a pesar de los riesgos de un mundo inseguro. Ellos lo saben y por eso visualizan sus proyectos como realizables en un futuro. La sociedad y el Estado, con sus políticas educativas y de formación en oficios, están ahí para amortiguar las fluctuaciones naturales de una sociedad multicultural y reducir los riesgos de la individualización moderna.

\section{Conclusiones}

Los procesos que aluden a la construcción de la identidad juvenil en las sociedades urbanas contemporáneas pueden ser mejor descritos y comprendidos bajo la categoría de la individualización, la cual alude a un sujeto que en condicio- 
nes de vulnerabilidad y riesgo social constantes se enfrenta solo a la desafiante tarea de conformarse como individuo.

De ahí que investigar transculturalmente cómo los adolescentes de diversos contextos culturales transitan dicha etapa de sus vidas resulta particularmente interesante, pues se destaca el papel que juegan las instituciones -y la sociedad en su conjunto- en tales procesos.

Es decir: conociendo la problemática de otros contextos sociales se podrían prever acciones concertadas con la participación de diversos agentes de la sociedad para gestionar programas sustentables que les brindaran asideros para el desarrollo de sus proyectos personales durante esta etapa; abrir nuevos espacios de participación social a los jóvenes para que sean ellos mismos los que orienten y construyan -con apoyo de los adultos y autoridades- su propio camino hacia el logro de su identidad.

En los dos casos analizados (grupo 1 y grupo 2) se encontraron distinciones, pero también similitudes que hacen referencia a la manera en que estos adolescentes se conciben a sí mismos y enfrentan diferenciadamente las demandas de la sociedad en que viven durante sus procesos de individualización. La expresión y orientación que tuvieron esos procesos cobró sentido y relevancia a la luz del trasfondo sociocultural en donde estos jóvenes se ubican. Las siguientes categorías fueron de utilidad para describir tales procesos en ambos grupos: a) negociación, b) agencia, c) representaciones y d) reflexividad.

Del análisis de los discursos se desprende que los jóvenes del grupo de Bremen siguen manteniendo (no obstante la transformación de su sociedad a la modernidad reflexiva) algunos valores tradicionales de su cultura, tales como la disciplina, el trabajo, la competitividad y el buen comportamiento social.

Se comentó anteriormente que tal permanencia de valores se debe a la función reforzadora de la escuela, las familias 
y a la sociedad en general, ya que representan la base del progreso en que se sustenta el país desde la mitad del siglo pasado. En ese sentido se puede afirmar que estos jóvenes, en cierta medida, han subjetivado las expectativas de un futuro promisorio que les ofrecen las instituciones de una sociedad que posee una de las economías más sólidas de la UE.

Por su parte, los relatos del grupo de Guadalajara son un tanto ambivalentes en cuanto al papel que juegan las instituciones y el Estado en la búsqueda de alcanzar sus proyectos de vida; para ellos, la escuela y su vida cotidiana pertenecen a mundos antagónicos en donde el imperativo familiar les obliga, en muchos casos, a continuar.

La escuela igualmente representa en el discurso un factor de oportunidades para el futuro, pero se advierte que el esfuerzo y disciplina sostenida que esta les demanda a los adolescentes rebasa su deseo o necesidad de logro personal. El éxito social, por lo tanto, parece no tener una clara vinculación con sus aprendizajes en esta etapa, de ahí que los hobbies, las relaciones sociales con los pares y el ocio les absorban la mayor parte de su tiempo libre. Al parecer, sus creencias de autoeficacia personal (seguridad de ser capaces de realizar algo) las construyen en función de las condiciones de seguridad y confianza que les brindan la familia e instituciones, de lo que estas les ofrezcan para un mejor mañana (trabajo, seguridad social, salud, estudios).

En ambos grupos de jóvenes se registraron diversas tonalidades en las construcciones de mundos de vida divergentes, que son reflejo internalizado de sus culturas, de sus valores, de sus marcos normativos, pero también de la importancia que tienen estos como pilares fundamentales de su desarrollo en una sociedad.

En esta investigación se confirma que las condiciones socioculturales (familia, escuela, amigos y espacios de convivencia) que prevalecen en ciertos contextos juegan un 
papel preponderante para encauzar los procesos de individualización juvenil que conducen a los sujetos a la adultez, permitiéndoles, en algunos casos, reducir las incertidumbres de la vida contemporánea y estimular la necesaria exploración en ambientes seguros para la configuración de sus proyectos personales.

Mientras en algunas sociedades y culturas se enfatiza más el diálogo a través de la negociación como estrategia para el logro de acuerdos y consensos con otros, como fue el caso del grupo 2, donde el contacto y convivencia multicultural se favorecen como parte de la vida cotidiana, en otras, como en el caso del grupo 1, el desarrollo de tales competencias sigue siendo una asignatura pendiente (y urgente) para formar ciudadanos participativos y críticos que consoliden el proceso democrático de un país como México.

En cuanto a la capacidad de reflexividad en ambos casos, cobra aquí relevancia el acceso y utilización de las fuentes de información, donde los jóvenes se retroalimentan para tomar decisiones, emprender iniciativas y plantear demandas a la sociedad. En el grupo 1, la utilización de los medios de comunicación e internet juega un papel cada vez más decisivo para formar su opinión e imaginar sus proyectos personales, sin dejar por ello de depender del soporte familiar cuando se trata de tomar decisiones importantes sobre la escuela. Es igualmente notorio en este grupo el surgimiento de una conciencia ecológica y social que coloca los temas del medio ambiente dentro de sus intereses y preocupación principales.

Una sociedad que ofrece estabilidad, pero también apertura en las necesidades de exploración y expresión juvenil, que da acceso a espacios de aprendizajes diversificados, a las artes y deportes, estará en condiciones de fortalecer los procesos de individualización en los jóvenes, mismos que son necesarios para enfrentar los retos de una sociedad cada vez más compleja e imprevisible como en la que hoy 
vivimos. Sobre todo, brindaría a los adolescentes mexicanos las condiciones necesarias para poner en práctica su capacidad de agencia en la construcción de sus propios proyectos personales.

Ayala, S. (200I). "La deserción escolar: ¿un callejón sin Bibliografía salida?”, en E. Matute, y R. M. Romo (coords.), Los retos en la educación del siglo XXI (Pp. 77-IOI). Guadalajara: U. de G.

Banco Mundial (s/f). Gasto en investigación y desarrollo (\% del PIB). Recuperado de: http://datos.bancomundial.org/ indicador/GB.XPD.RSDV.GD.ZS

Beck, U. (2002). La sociedad del riesgo global. Madrid: Siglo $X X I$.

y Beck-Gernsheim, E. (2002). Institutionalized Individualism and its Social and Political Consequences. Londres: Sage.

Giddens, A., y Lash, S. (1997). La modernización reflexiva. Políticas, tradición y estética en el orden social moderno. Buenos Aires: Alianza.

Beck-Gernsheim, E. (2000). Was kommt nach der Familie?: Einblicke in nuen Lebensformen. Alemania: Taschenbuch. Brater, M. (2002). "Escuela y formación bajo el signo de la individualización”, en U. Beck (comp.), Los hijos de la libertad (Pp. I26-I5I). México: Fondo de Cultura Económica.

Briceño, G. (20I I). “Deserción escolar y migración: correlatos de la realidad mexicana”, en G. Briceño (coord.), Migración: desafios y posibilidades (pp. 85-99). México: DAAD, Goethe Institut y APERFA.

(2012a). Entrevista personal realizada a Inge, adolescente de 14 años. Bremen, Alemania.

(20l2b). Entrevista personal realizada a Viktoria, adolescente de 17 años. Bremen, Alemania. 
Bibliografía

(20I2c). Entrevista personal realizada a Klaus, adolescente de 14 años. Bremen, Alemania. (2012d). Entrevista personal realizada a Magdalena, adolescente de 17 años. Bremen, Alemania. (20I2e). Entrevista personal realizada a María, adolescente de 13 años. Bremen, Alemania.

(20I2f). Entrevista personal realizada a Helen, adolescente de 14 años. Bremen, Alemania.

( $2012 \mathrm{~g})$. Entrevista personal realizada a Inke, adolescente de 13 años. Bremen, Alemania.

Datenreport (20I4). Datenreport. Der Stiftung Weltbevölkerung. Soziale und demographische daten weltweit. Recuperado de: http://www.weltbevoelkerung.de/fileadmin/ content/PDF/Datenreport_20I4_Stiftung_Weltbevoelkerung.pdf

Deutschland Magazin (s/f). "Gesellschaft \& Integration". Recuperado de: https:/www.deutschland.de/de/topic/ leben/gesellschaft-integration

Diario Oficial de la Federación (30 de abril de 2014). Programa Nacional de Juventud 2014-20/8. México: Segob.

Die Welt (0I de agosto de 20I3). "Das Geld hält Deutsche vom Kinderkriegen ab". Recuperado de: http://www. welt.de/politik/deutschland/article I I 8587583/DasGeld-haelt-Deutsche-vom-Kinderkriegen-ab.html

Dubet, F. (2006). El declive de la institución. Profesiones, sujetos e individuos en la modernidad. Barcelona: Gedisa.

Fundación Mexicana para el Fomento de la Lectura, A. C. (20I2). Encuesta Nacional de Lectura 2012. México: FunLectura.

Instituto Mexicano de la Juventud (20/2). Encuesta Nacional de Valores en Juventud. México: UNAM, IMJUVE. Recuperado de: www.imjuventud.gob.mx/imgs/uploads/ ENVAJ_20I2.pdf

Kroger, J. (2003). "Identity development during adolescence”, en G. R. Adams, y M. D. Berzonsky (eds.), 
Blackwell handbook of adolescence (pp. 205-226). Malden: Blackwell.

Lash, S. (2003). "Reflexivity as Non-Linearity". Theory, Culture \& Society, 20(2), 49-58.

Marcia, J. E. (1993). "The relational roots of identity", en J. Kroger (ed.), Discussions on ego identity (pp. 10I-120). Hillsdale: Erlbaum.

Mertens, D. (2005). Research and evaluation in Education and Psychology: Integrating diversity with quantitative, qualitative, and mixed methods. Thousand Oaks: Sage.

Morduchowicz, R. (2004). El capital cultural de los jóvenes. Argentina: FCE.

Meza, G. L., y Pederzini, V. C. (2008). “Migración internacional y escolaridad como medios alternativos de movilidad social: el caso de México". Estudios Económicos, (número extraordinario), 163-206.

Papalia, D. E., Wendkos, S. O., y Feldman, R. D. (20I0). Desarrollo humano. México: Interamericana Mc Graw Hill Editores.

Reyes, J. A. (2009). Adolescencia entre muros. Escuela secundaria y la construcción de identidades juveniles. México: FLACSO.

Ruiz, M. E., y Briceño, G. (2005). "La interdisciplinariedad en los estudios de la subjetividad: nuevos paradigmas en las ciencias sociales", en E. M. Ruiz (coord.), Interdisciplinariedad (pp. 19-39). Guadalajara: Universidad de Guadalajara/DEES.

OCDE (2013). Education at a Glance 2013. Indicadores y anexos. Tabla Cl.I ${ }^{a}$. Recuperado de: http://www.oecd. org/edu/educationataglance20 I3-indicatorsandannexes. htm\#ChapterC

Schmaus, S. (20I4). "Wie leben junge Menschen in Deutschland?". DW. Recuperado de: http://www.dw.com/de/ wie-leben-junge-menschen-in-deutschland/a- 18077083 
Bibliografía
Stake, R. E. (2000). “Case studies”, en N. K. Denzin, y Y. S. Lincoln (eds.), Handbook of qualitative research (pp. 435-453). Thousand Oaks: Sage.

Tiramonti, G. (2004). "Una nueva cartografía de sentidos para la escuela”, en G. Tiramonti (comp.), La trama de la desigualdad educativa: mutaciones recientes en la escuela media (Pp. I0I-I I8). Buenos Aires: Manantial.

Twenge, J. M., y Campbell, W. K. (2009). The Narcissism Epidemic: Living in the Age of Entitlement. Nueva York: Atria Paperback.

Villalpando, M. E., Briceño, G., Castañeda, M. G., y Elizarrarás, P. (20I2). Consumo y cambio cultural en estudiantes de secundaria en Jalisco. México: CONACYT, SEJ.

Wikipedia (s/fa). "Bremen". Recuperado de: https:// es.wikipedia.org/wiki/Bremen\#/media/File:Germany_ location_map.svg (s/fb). "Mapa de México con nombres”. Recuperado de: https://es.wikipedia.org/wiki/Plantilla:Mapa de_M\%C3\%A9xico_con_nombres\#/media/File:Blank_ map_of_Mexico.svg

Yin, R. (1994). Case Study Research: Design and Methods. Thousand Oaks: Sage Publications. 